Observaciones de movimientos de murciélagos en un

paisaje fragmentado en el Istmo de Tehuantepec, México

\title{
Notes on bat movements in a fragmented landscape in the Tehuantepec Isthmus, Mexico
}

\author{
Mario C. Lavariega ${ }^{1 *}$ and Miguel Briones-Salas ${ }^{1}$
}

\begin{abstract}
${ }^{1}$ Laboratorio de Vertebrados Terrestres (Mastozoología), Centro Interdisciplinario de Investigación para el Desarrollo Integral Regional, Unidad Oaxaca, Instituto Politécnico Nacional. Hornos 1003, Colonia Noche Buena. Santa Cruz Xoxocotlán 71230. Ciudad de Oaxaca, Oaxaca, México. E-mail: mariolavnol@yahoo.com.mx (MCL); miguelbrionessalas@hotmail.com (MBS). ${ }^{*}$ Corresponding author
\end{abstract}

Several studies have observed that bat species respond differently to landscape changes and that individuals tend to expand their home range when the habitat is not optimal. Tropical dry forest in the Isthmus of Tehuantepec, Mexico, shows a high habitat loss and fragmentation by anthropogenic causes. However, how bats use this fragmented landscape is unknown. Therefore, the aim of this study was to describe the temporal and spatial movements of bats fitted with radio transmitters in a fragmented region of the Isthmus of Tehuantepec. In July 2011, six bats belonging to three species were captured: an adult female of Artibeus lituratus, a juvenile female of $A$. jamaicensis, an adult male of Leptonycteris yerbabuenae (Family Phyllostomidae), and three adult females of Pteronotus parnellii (Family Mormoopidae) were fitted with a radio transmitter. Bats were monitored during night (18:00 to 06:00 h) from fixed stations for 17 days. The activity was obtained by quantifying the frequency of bearings in intervals of one hour. Simultaneous bearings were triangulated for locations, which were exported to a vector map where type of land use and vegetation was determined. The home range was calculated using two techniques: minimum convex polygon and fixed Kernel. A total of 75 bearings corresponding to an adult female of A. lituratus ( $n=36,48.0 \%)$, three adults females of P. parnellii $(n=30,40.0 \%)$, and an adult male of $L$. yerbabuenae $(n=8 ; 10.6 \%)$ were obtained. A juvenile female of $A$. jamaicensis was located on one occasion (1.3\%). Female of A. lituratus showed activity between 19:00 and 23:00 h and between 24:00 and 05:00 h, with a peak between 03:00 y 04:00 h. This bat widely used the area with preference to riparian vegetation. Based on their locations, a home range (Kernel $95 \%$ ) of 8,394 hectares and a minimum convex polygon of 2,587 hectares were estimated. The male of $L$. yerbabuenae showed activity only between $22: 00$ and $02: 00 \mathrm{~h}$ and their locations were on agriculture land $(n=2)$. The observed activity of $P$. parnellii began at 20:00 h, it was increased to at 22:00 h (one activity peak between 01:00 and 3:00 h) and gradually declined until 06:00 h. The three females of $P$. parnellii were located in agricultural land $(n=4)$ and cultivated pasture $(n=1)$. The home range of $A$. lituratus observed in the present study is the largest reported for phyllostomid bats, possibly in response to landscape condition. Activity and locations of three females of P. parnellii and a male of $L$. yerbabuenae suggest that they occasionally use the study area in their way to more productive areas.

Se ha observado que las especies de murciélagos responden de manera diferencial a los cambios en el paisaje y que los individuos tienden a ampliar su ámbito hogareño cuando el hábitat no es óptimo. Las selvas secas de la Planicie costera del Istmo de Tehuantepec, en México, presentan una alta pérdida y fragmentación por causas humanas. Sin embargo, se desconoce cómo los murciélagos usan este paisaje fragmentado. Por lo anterior, el objetivo de este estudio fue describir por primera vez los movimientos temporales y espaciales de murciélagos equipados con radio-transmisores en una zona fragmentada del Istmo de Tehuantepec. En julio de 2011 se capturaron seis murciélagos de tres especies: una hembra adulta de Artibeus lituratus, una hembra joven de A. jamaicensis, un macho adulto de Leptonycteris yerbabuenae (Familia Phyllostomidae) y tres hembras adultas de Pteronotus parnellii (Familia Mormoopidae), a los que se les adhirió un radio-transmisor. Durante 17 días de estudio, los murciélagos se buscaron a lo largo de la noche (18:00 a 06:00 h) desde estaciones fijas. La actividad se obtuvo al cuantificar la frecuencia de lecturas del rumbo en intervalos de una hora. Las lecturas de rumbo simultáneas fueron trianguladas para obtener puntos geográficos, mismos que se exportaron a un mapa vectorial en donde se determinó la cobertura de uso de suelo y vegetación. El área del ámbito hogareño se calculó mediante dos técnicas: polígono mínimo convexo y Kernel fijo. Se obtuvieron un total de 75 lecturas del rumbo, correspondientes a una hembra adulta de $A$. lituratus $(n=36,48.0 \%)$, tres hembras adultas de $P$. parnellii $(n=$ $30 ; 40.0 \%)$ y un macho adulto de $L$. yerbabuenae $(n=8 ; 10.6 \%)$. Una hembra joven de $A$. jamaicensis se localizó en una 
sola ocasión (1.3 \%). La hembra de A. lituratus mostró actividad entre las 19:00 y 23:00 h y entre las 24:00 y 05:00 h, con un pico entre las 03:00 y 04:00 h. Este murciélago usó ampliamente la zona con preferencia hacia vegetación ribereña. Con base en los puntos geográficos se le calculó un ámbito hogareño (Kernel 95 \%) de 8,394 hectáreas y un polígono mínimo convexo de 2,587 hectáreas. Del macho de $L$. yerbabuenae solo se observó actividad entre las 22:00 y 02:00 h y las localizaciones estuvieron en áreas para agricultura $(n=2)$. El horario de actividad observado de $P$. parnellii comenzó a las 20:00 h, se incrementó hacia las 22:00 h (un pico de actividad entre las 01:00 y 3:00 h) y disminuyó gradualmente hasta las 06:00 h. Las tres hembras de $P$. parnellii se ubicaron en áreas agrícolas $(n=4)$ y en pastizal cultivado $(n=1)$. El ámbito hogareño de $A$. lituratus observado en el presente estudio es uno de los más grandes reportados para murciélagos filostómidos, posiblemente como respuesta a la condición del paisaje. Los puntos geográficos y horas de actividad de tres hembras de P. parnellii y de un macho de $L$. yerbabuenae sugieren que utilizan la zona ocasionalmente en su paso a otras áreas más productivas.

Key words: Artibeus jamaicensis, A. lituratus, home range, Leptonycteris yerbabuenae, Pteronotus parnellii, radiotelemetry, wind farms.

C 2016 Asociación Mexicana de Mastozoología, www.mastozoologiamexicana.org

\section{Introducción}

La pérdida y fragmentación del hábitat trae consigo efectos en la biodiversidad, como cambios en la composición de las comunidades, en las interacciones intra e interespecíficas y en la conducta de los individuos (Fahrig 2003). Uno de los grupos más empleados para evaluar estos los disturbios, son los murciélagos, debido a que desempeñan un papel importante en la estructura y función de los ecosistemas, son abundantes y relativamente fáciles de estudiar (Erkert 2000, Patterson et al. 2003). En general, se ha observado que las especies de murciélagos responden de manera diferencial a la condición del paisaje (Albrecht et al. 2007) y que los individuos tienden a ampliar su ámbito hogareño cuando el hábitat no es óptimo (Aihartza et al. 2003, Goiti et al. 2003, Bianconi et al. 2006, Cortés-Delgado y Sosa 2014).

Las selvas secas de la Planicie costera del Istmo de Tehuantepec, Oaxaca, en el sur de México, son ecosistemas con un alto impacto antropogénico por la pérdida y fragmentación del hábitat, principalmente por la agricultura, ganadería y establecimiento de parques eólicos (Padilla y Quiroz 2005, Carrillo-Reyes et al. 2012, Lorenzo et al. 2014). Sin embargo, se desconoce cómo los murciélagos usan este paisaje fragmentado. Por lo anterior, el objetivo de este estudio fue describir por primera vez, los movimientos, actividad y el ámbito hogareño de murciélagos equipados con radio-transmisores en una zona fragmentada del Istmo de Tehuantepec, Oaxaca, México.

\section{Material y Metodos}

Área de estudio. Se localiza en el municipio de Juchitán de Zaragoza $\left(16^{\circ} 30^{\prime}\right.$ y $16^{\circ} 36^{\prime} \mathrm{N},-94^{\circ} 46^{\prime}$ y $-94^{\circ}$ $53^{\prime} \mathrm{W} ; 25 \mathrm{~m}$ ), en la subprovincia fisiográfica Planicie costera de Tehuantepec (Ortiz-Pérez 2004), sureste del estado de Oaxaca, México (Figura 1a). El clima predominante es el cálido subhúmedo (A (wo)), con una temperatura media anual mayor de $22^{\circ} \mathrm{C}$ y la temperatura del mes más frío mayor de $18^{\circ} \mathrm{C}$. La precipitación media anual es de 1,000 mm, la precipitación del mes más seco se encuentra entre 0 y $60 \mathrm{~mm}$ y el porcentaje de lluvia invernal es del 5 al $10.2 \%$ del total anual (García 2001). La zona de estudio ha sido modificada para actividades productivas primarias, predominando la agricultura de riego, agricultura de temporal con cultivos anuales y pastizal cultivado. Existen remanentes de vegetación halófila y gipsófila, selva baja caducifolia y subcaducifolia con vegetación secundaria y vegetación de galería a lo largo del río Espíritu Santo (INEGI 2005). El Istmo de Tehuantepec tiene un alto potencial para la generación de energía eólica, mismo que se está aprovechando con la operación de parques eólicos (Hernández-Escobedo et al. 2010). 


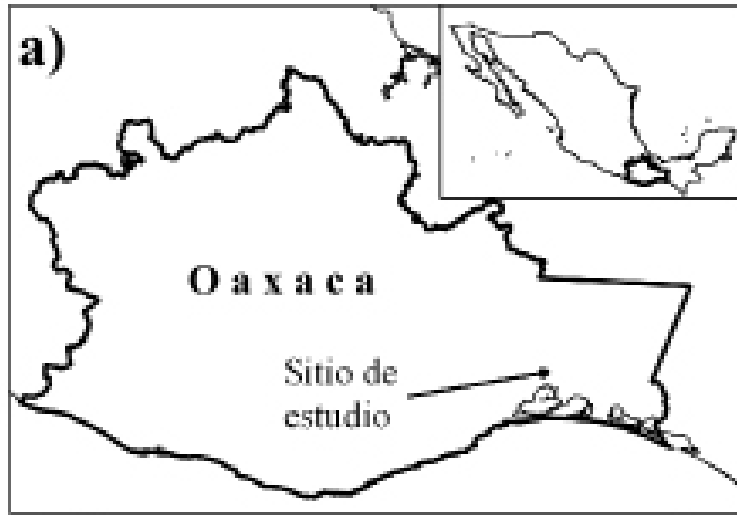

Lejenda

- Riog

$\Longrightarrow$ Canales de riego

- - carreteras

Uso de suclo y vegetación

Selva baja caducifolia

Selva baja caducifolia con veg. sec

Selva baja caducifolia espinosa con veg sec. Pastizal

Agricultura de riego

Agricultura de temporal

A sentamiento humano
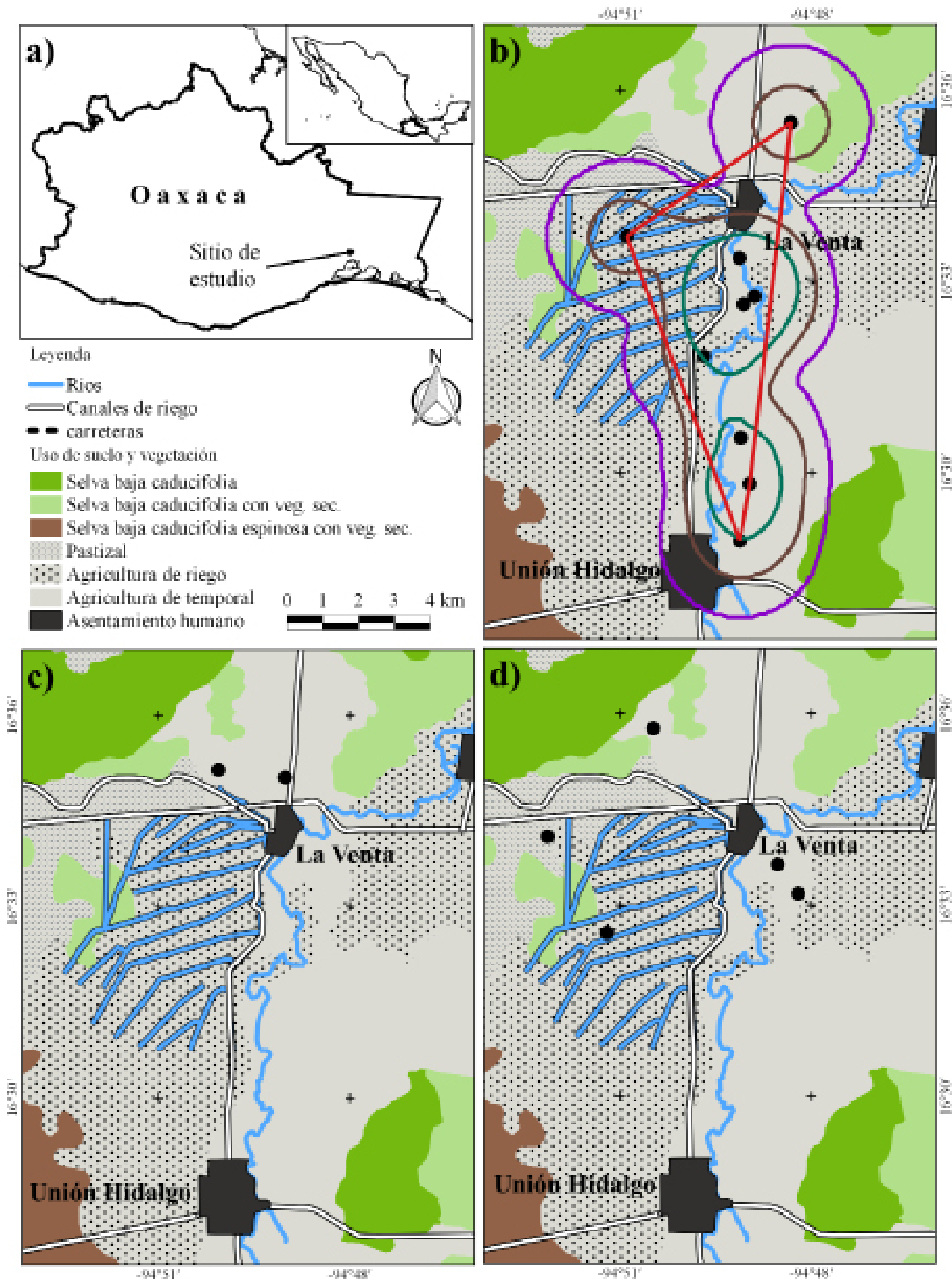

Figura 1. Localizaciones de murciélagos equipados con radio-transmisores en el Istmo de Tehuantepec, México. a) Macrolocalización. b) Hembra adulta de Artibeus lituratus: puntos negros, localizaciones; ámbito hogareño: polígono turquesa, 50 \%; polígono café oscuro, $75 \%$; polígono morado, 95 \%; polígono mínimo convexo: polígono rojo. c) Macho adulto de Leptonycteris yerbabuenae: puntos negros, localizaciones. d) Hembras adulta de Pteronotus parnellii: puntos negros, localizaciones. 
Captura y radio-telemetría. Entre el 3 y 6 de julio de 2011 se capturaron seis murciélagos, de cuatro especies y dos familias (Tabla 1), con redes de niebla de $12 \times 2.5 \mathrm{~m}$ desplegadas a una altura mínima de $1.50 \mathrm{~m}$ del suelo y ubicadas entre caminos y vegetación aledaña a cuerpos de agua. Los murciélagos fueron colocados en bolsas de manta; posteriormente se les tomaron medidas somáticas, se pesaron y fueron determinados a nivel de especie con claves especializadas (Álvarez et al. 1994, Medellín et al. 1997). La determinación del sexo y condición reproductiva de los murciélagos se clasificó de acuerdo a Kunz et al. (1996). La edad se determinó con base en el desgaste de la dentadura y la osificación de las falanges, clasificándolos como joven o adulto.

A cada murciélago se le cortó a ras una sección del pelaje de la región dorsal entre las escápulas y se adhirió un radio-transmisor (Modelo TXB-001G; Telenax, Quintana Roo, México) con pegamento hipodérmico. Los radio-transmisores contaban con una vida de batería de al menos 14 días y un peso de $0.6 \mathrm{~g}$, lo que significó un peso menor al $5 \%$ del peso de los murciélagos (Aldridge y Brigham 1988). Los murciélagos fueron mantenidos en cautiverio por 20 minutos, durante los cuales se observó su estado, se les proporcionó agua y se verificó que el dispositivo estuviera firmemente adherido; finalmente, fueron liberados en el mismo sitio de colecta. Las capturas se realizaron bajo el amparo de permiso de colector (FAU-007) emitido por la Secretaria de Medio Ambiente y Recursos Naturales (SEMARNAT).

En el área de estudio se ubicaron estaciones de monitoreo fijas en sitios elevados (e. g. montículos de tierra, puentes) y evitando fuentes de interferencia radio-magnética (e.g. torres de transmisión eléctrica, maquinaria). Cada noche se trabajó en dos estaciones que contaban con una antena bidireccional Yagui de tres elementos (RX-TLNX; Telonics, AZ), un receptor de radio-telemetría (R100; Communications Specialists, CA) y un radio de alto alcance (Kenwood, Japón) para mantener contacto entre estaciones. El estudio se realizó durante 17 días (del 3 al 20 de julio), iniciando a partir de las 19:00 hasta las 06:00 h del día siguiente. Durante este periodo se efectuó la búsqueda de los radio-transmisores en lapsos de una hora; en todo momento se mantuvo contacto entre las estaciones para aumentar la posibilidad de obtener lecturas de rumbo simultáneas. Una vez localizado algún radio-transmisor, se obtuvo el rumbo con la ayuda de un clinómetro. Se llevó un registro de la geoposición de cada estación, así como la hora, lecturas del rumbo e intensidad de la señal.

Análisis de datos. Las lecturas del rumbo fueron exportadas al software Locate 3.33 (Nams 2000) donde se calculó por medio de triangulación, la probable localización geográfica del transmisor colocado en cada uno de los murciélagos en el sistema de coordenadas geográficas decimales (Datum y elipsoide WGS84). Las localizaciones geográficas trianguladas obtenidas de Locate 3.33, fueron exportadas a Arc View 3.2 (ESRI, Redlands, California) en donde se calculó, con la extensión Home Range (Rogers y Kie 2007), el área del ámbito hogareño usando dos técnicas: Polígono Mínimo Convexo al $100 \%$ y Kernel fijo al 50 y al $95 \%$ con un cálculo ad hoc del parámetro ejecutado (Loayza y Loiselle 2008). Finalmente, los puntos geográficos se sobrepusieron en una capa vectorial de uso de suelo y vegetación (INEGI 2005), en donde se calculó el porcentaje de las clases de cada tipo de cobertura. La actividad de los individuos capturados, se estimaron considerando el horario de la primera y la última lectura de rumbo, con ello, se estimó un periodo en horas de actividad fuera de los refugios. Cabe aclarar que el registro de la actividad estuvo limitado por el intervalo de alcance de los radiotransmisores.

\section{Resultados}

En 17 días de seguimiento se obtuvieron un total de 75 lecturas del rumbo, correspondientes a una hembra adulta de Artibeus lituratus ( $n=36,48.0 \%$ ), tres hembras adultas de Pteronotus parnellii ( $n=30 ; 40.0 \%$ ) y un macho adulto de Leptonycteris yerbabuenae $(n=8 ; 10.6 \%)$. Una hembra 
joven de $A$. jamaicensis se localizó en una ocasión (1.3\%). El promedio de días con lecturas del rumbo fue de 6.3, con un intervalo de un día para A. jamaicensis y 14 para A. lituratus. Del total de lecturas del rumbo, solo en 32 lecturas simultáneas (42.6 $\%)$ fue posible triangularlas en Locate para obtener las coordenadas de localización geográfica de cada individuo marcado. Debido a la baja cantidad puntos geográficos de los individuos de A. jamaicensis, P. parnellii y L. yerbabuenae, no fue posible calcular su ámbito hogareño (Tabla 1).

Hembra adulta de Artibeus lituratus. Ocupó ampliamente la zona, a excepción del área urbana, se movió a través de la matriz de campos de cultivo, canales de riego y cerca del río Espíritu Santo. De nueve puntos geográficos, siete (87.5 \%) se encontraron a lo largo del río Espíritu Santo y uno más, muy cerca de un canal de riego (Figura 1b). Se observó actividad a lo largo de la noche, con tres picos: entre las 20:00 y 21:00 h, las 02:00 y 05:00 h y entre las 06:00 y 07:00 h (Figura 2).

Esta hembra se localizó en dos ocasiones: el 5 de julio, primero a las 20:45 h y posteriormente a las 24:05 h; con ello se calculó una distancia mínima recorrida de $5.4 \mathrm{~km}$. Con base en los puntos geográficos se estimó, con Kernel al $95 \%$, un ámbito hogareño en 8,394 ha, con una superficie del área central (50\%) de 1,507 ha y un polígono mínimo convexo de 2,587 ha (Tabla 2, Figura 1b).

Macho adulto de Leptonycteris yerbabuenae. Este macho, fue uno de los individuos con el menor número de localizaciones geográficas efectivas (trianguladas), de ocho lecturas de rumbo obtenidas, solo dos fue posible triangularlas y fueron ubicados por triangulación en campos agrícolas (Figura 1c). Se registró un periodo corto de actividad, en el intervalo de alcance de los transmisores, entre las 22:00 y 02:00 $\mathrm{h}$ (Figura 3).

Tres hembras adultas de Pteronotus parnellii. Los puntos geográficos se ubicaron en áreas agropecuarias (zonas agrícolas $n=4$; pastizal cultivado $n=1$ ); las 25 lecturas de rumbo restantes, no fue posible triangularlas, perdiendo con ello el $60 \%$ de los datos obtenidos en campo. El horario de actividad observado para las hembras de P. parnellii comenzó a las 20:00, se incrementó hacia las 22:00 h (un pico de actividad entre las 01:00 y 3:00 h) y disminuyó gradualmente hasta las 06:00 h (Figura 4).

Tabla 1. Especies de murciélagos equipados con radio-transmisores en el Istmo de Tehuantepec.

\begin{tabular}{|c|c|c|c|c|c|c|c|}
\hline $\begin{array}{l}\text { Familia } \\
\text { Especie }\end{array}$ & Frecuencia & $\begin{array}{c}\text { Gremio } \\
\text { alimenticio }\end{array}$ & Sexo & Edad & $\begin{array}{l}\text { Longitud } \\
\text { del } \\
\text { antebrazo } \\
(\mathrm{mm})\end{array}$ & $\begin{array}{c}\text { Masa } \\
\text { (gr) }\end{array}$ & $\begin{array}{c}\text { Número de } \\
\text { noches con } \\
\text { lecturas del } \\
\text { rumbo }\end{array}$ \\
\hline \multicolumn{8}{|l|}{ Mormoopidae } \\
\hline Pteronotus parnellii & 150.103 & Insectívoro & Hembra & Adulto & 59 & 30 & 8 \\
\hline Pteronotus parnellii & 150.142 & Insectívoro & Hembra & Adulto & 55 & 35 & 5 \\
\hline Pteronotus parnellii & 150.183 & Insectívoro & Hembra & Adulto & 58 & 26 & 5 \\
\hline \multicolumn{8}{|l|}{ Phyllostomidae } \\
\hline Leptonycteris yerbabuenae & 150.222 & Nectarívoro & Macho & Adulto & 55 & 43 & 5 \\
\hline Artibeus lituratus & 150.062 & Frugívoro & Hembra & Adulto & 65 & 63 & 16 \\
\hline Artibeus jamaicensis & 150.662 & Frugívoro & Hembra & Joven & 61 & 49 & 1 \\
\hline
\end{tabular}




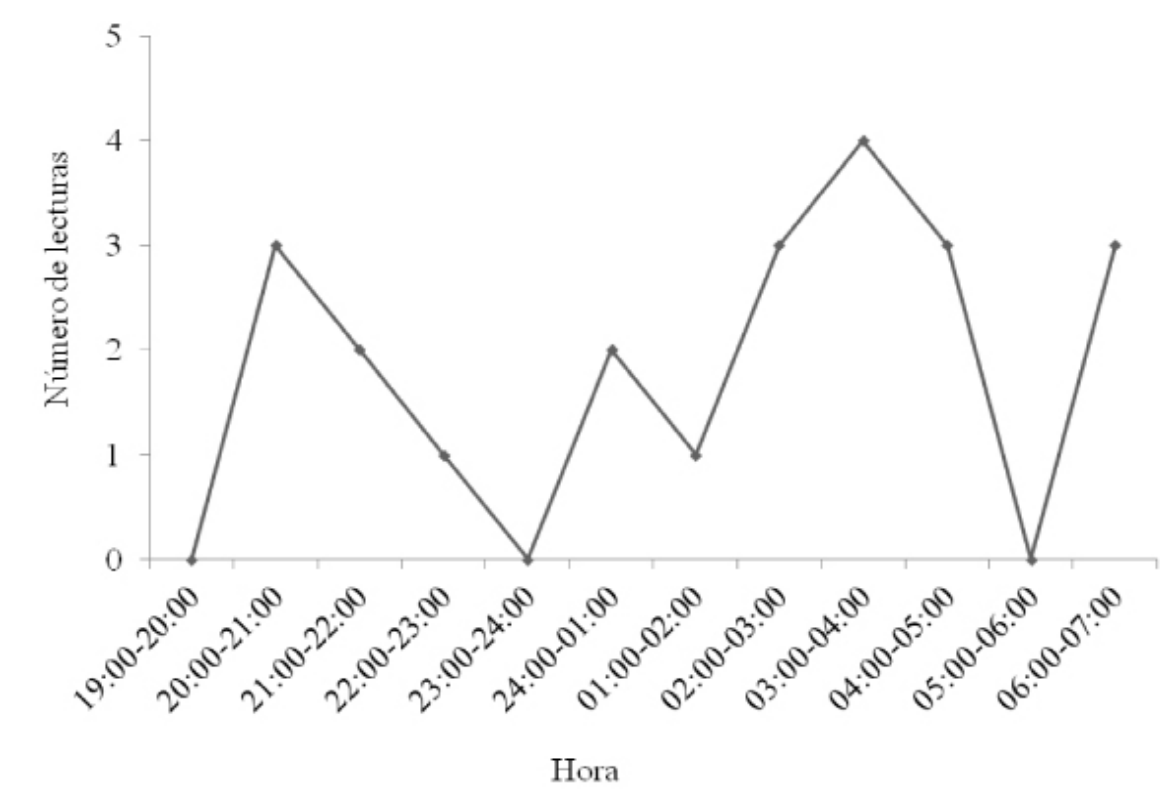

Figura 2. Actividad circadiana observada a traves de lecturas del rumbo de una hembra adulta de Artibeus lituratus equipada con radio-transmisor en el Istmo de Tehuantepec.

\section{Discusión}

La cantidad de lecturas del rumbo en estudios de murciélagos suele ser baja debido al constante movimiento de los animales (Handley y Morrison 1991). En el presente trabajo, menos de la mitad de las lecturas del rumbo fueron simultáneas, lo que se reflejó en la baja cantidad de puntos geográficos obtenidos por individuo. En otros estudios, para solventar este aspecto se ha estimado la distancia desde el receptor al transmisor con la calibración de la fuerza de la señal, una alternativa poco útil en ambientes con alta perturbación antrópica y fuentes de interferencia electromagnética, como lo realizado en el presente estudio.

Los patrones de actividad observados en el presente trabajo no significan que los individuos estuvieron más activo a estas horas, si no que fue posible localizarlos en estos periodos de tiempo desde las estaciones de radiolocalización. Con respecto a Artibeus lituratus, se observó un patrón de actividad de aproximadamente $10 \mathrm{~h}$ (20:00 $\mathrm{h} \sim$ 06:00 h del siguiente día), con periodos de localización entre las 20:00 y 21:00 h, entre las 02:00 y 05:00 h y entre las 06:00 y 07:00 h. Este patrón de actividad polimodal fue muy similar al reportado para la misma especie mediante la frecuencia de capturas en redes de niebla en un bosque ripario en Tamaulipas, México (Arriaga-Flores et al. 2012). Es posible que el individuo de A. lituratus presente el mismo comportamiento que $A$. jamaicensis en Barro Colorado, Panamá donde se observó que salen del refugio diurno $0.5 \mathrm{~h}$ después de la puesta del sol y retornan $0.5 \mathrm{~h}$ antes de la salida del sol (Handley y Morrison 1991).

Se ha observado también que $A$. lituratus recorre grandes distancias, como lo registrado en el sureste de Brasil, donde una hembra recorrió $113 \mathrm{~km}$ en un intervalo de altura de $738 \mathrm{~m}$ (Arnone et al. 2016). Anteriormente, Bernard y Fenton (2003) y Mendes et al. (2009) habían registrado estos grandes desplazamientos por parte de esta especie. Es altamente probable que los individuos de A. lituratus de la región del Istmo de Tehuantepec, en 
México, recorran grandes distancias en búsqueda de sitios de alimentación, dado que esta zona se encuentra fuertemente fragmentada y con poca cobertura vegetal. De igual forma, la poca cantidad de lecturas de rumbo obtenidas para esta especie y otras más, posiblemente también se deba a que en la zona se encuentran una gran cantidad de parques eólicos que ponen en riesgo su integridad física (SENER 2015).

Fenton et al. (2001) encontraron que individuos de $P$. parnellii salían de sus refugios entre las 18:15 y 18:30 h para dirigirse al área de bosque. En el presente estudio, tres hembras fueron detectadas entre las 20:00 y 21:00 h, tres horas después de lo reportado con anterioridad, alcanzando un pico entre las 01:00 a 04:00 h. Por lo anterior, y aunque no se midió la disponibilidad de alimento, es probable que los individuos de P. parnellii inicien actividad en áreas cercanas a sus refugios para posteriormente desplazarse a la zona de estudio en su paso a otros sitios con mayor disponibilidad de insectos y retornar a sus refugios antes del amanecer (04:00 h).

A lo largo del estudio dos individuos de P. parnellii fueron detectados frecuentemente en el área de estudio. Ésta especie muestra tolerancia a ambientes fragmentados (Estrada y Coates-Estrada 2001, Galindo-González 2004, Montiel et al. 2006). La presencia de vegetación resulta importante para su desplazamiento (Rydell et al. 2002, Estrada y Coates-Estrada 2001), debido a que forrajean a menos de un metro de la vegetación o dentro de ésta y raramente en áreas abiertas (Bonaccorso 1979, $\underline{\text { Herd 1983, Jennings et }}$ al. 2004). También A. jamaicensis y A. lituratus se consideran tolerantes a la perturbación (Galindo-González 2004); para la primera especie hubo una sola localización, lo que pudo deberse a fallas en el radio-transmisor o porque se trataba de un animal en dispersión o posiblemente migración (Arnone et al. 2016).

El murciélagos magueyero macho (L. yerbabuenae) registró un bajo número de puntos geográficos, y éstos se encontraron al norte, cerca de áreas con selva baja caducifolia, tipo de vegetación que se caracteriza por la presencia de plantas agaváceas y cactáceas, fuentes importantes de alimento de esta especie (Molina-Freaner 2004). En este sentido, murciélagos nectarívoros usan hasta dos terceras partes del contenido calórico de sus cuerpos cada noche (Von Helversen y Winter 2003), e invertir energía en un hábitat de calidad pobre no sería eficiente en términos de los costos-beneficios, por el elevado número de plantas que deben visitar (e.g. 80-100 flores de cactáceas; $\underline{\text { Horner }}$ et al. 1998, Von Helversen y Winter 2003, Voigt et al. 2006).

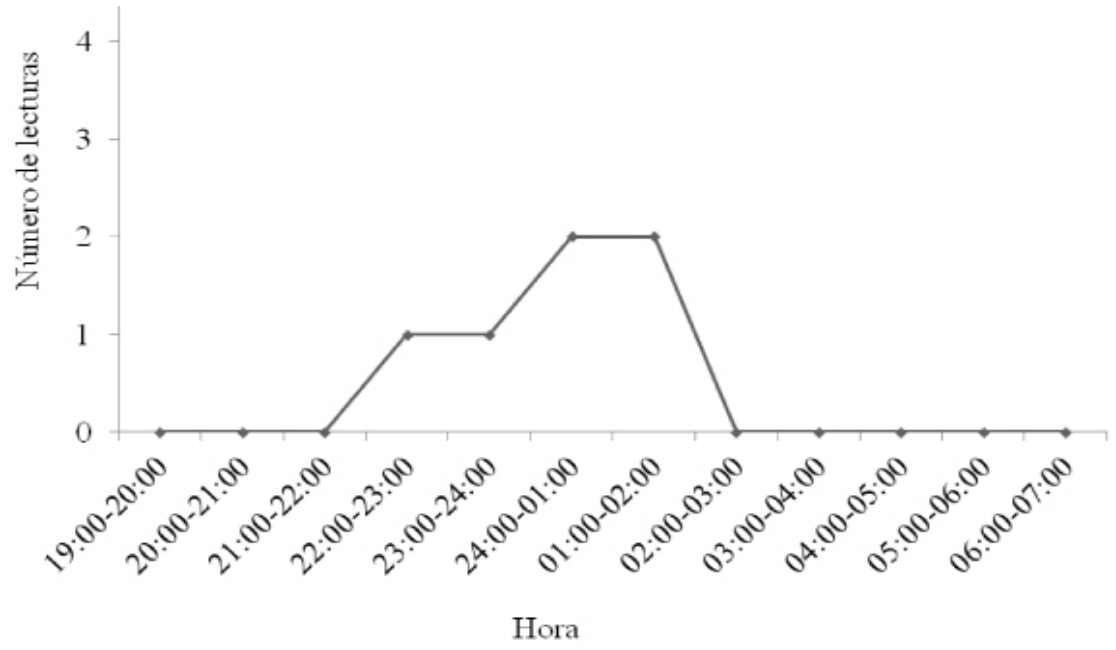

Figura 3. Actividad circadiana observada a traves de lecturas del rumbo de un macho adulto de Leptonycteris yerbabuenae equipado con radio-transmisor en el Istmo de Tehuantepec. 
El presente trabajo es de los pocos publicados con información del ámbito hogareño de quirópteros con telemetría en México. Horner et al. (1998), estudiaron los movimientos de Leptonycteris yerbabuenae en el Desierto de Sonora, México, aunque no analizaron el ámbito hogareño, registraron los movimientos por cerca de 30 km, desde la Isla Tiburón hasta el continente en busca de alimento. En la frontera entre Estados Unidos de América y México, Tibbitts et al. (2002), encontraron que tres murciélagos molósidos de la especie Eumops underwoodi tuvieron ámbitos hogareños grandes (9,962, 16,035 y 47,375 has) con actividad de forrajeo en hábitats naturales como transformados. Arias-Cóyotl et al. (2006) reportaron que tres individuos de L. yerbabuenae viajaron $15 \mathrm{~km}$ desde sus refugios hasta las áreas de alimentación en la Mixteca de Oaxaca. El estudio de Cortés-Delgado y Sosa (2014), analiza el ámbito hogareño de Sturnira hondurensis entre matrices con dominancia de bosque mesófilo de montaña y café de sombra en Veracruz. El ámbito hogareño varió para 26 individuos entre: 3.93-311.30 ha, promedio: 56.7 ha); sin embargo, no encontraron diferencias significativas entre los dos ambientes estudiados.

En el presente trabajo, tanto el ámbito hogareño, calculado con el Kernel al $95 \%$, como el polígono mínimo convexo de $A$. lituratus, fueron mayores en comparación los obtenidos con radio-telemetría en otros filostómidos frugívoros (Bernard y Fenton 2003, Bonaccorso et al. 2006, Albrecht et al. 2007, Loayza y Loiselle 2008, CortésDelgado y Sosa 2014; Tabla 2). Las causas que influyen en el área de movimiento de los murciélagos son la calidad y configuración del paisaje (Bernard y Fenton

Tabla 2. Área de actividad de murciélagos filostómidos equipados con transmisores de radio-telemetría.

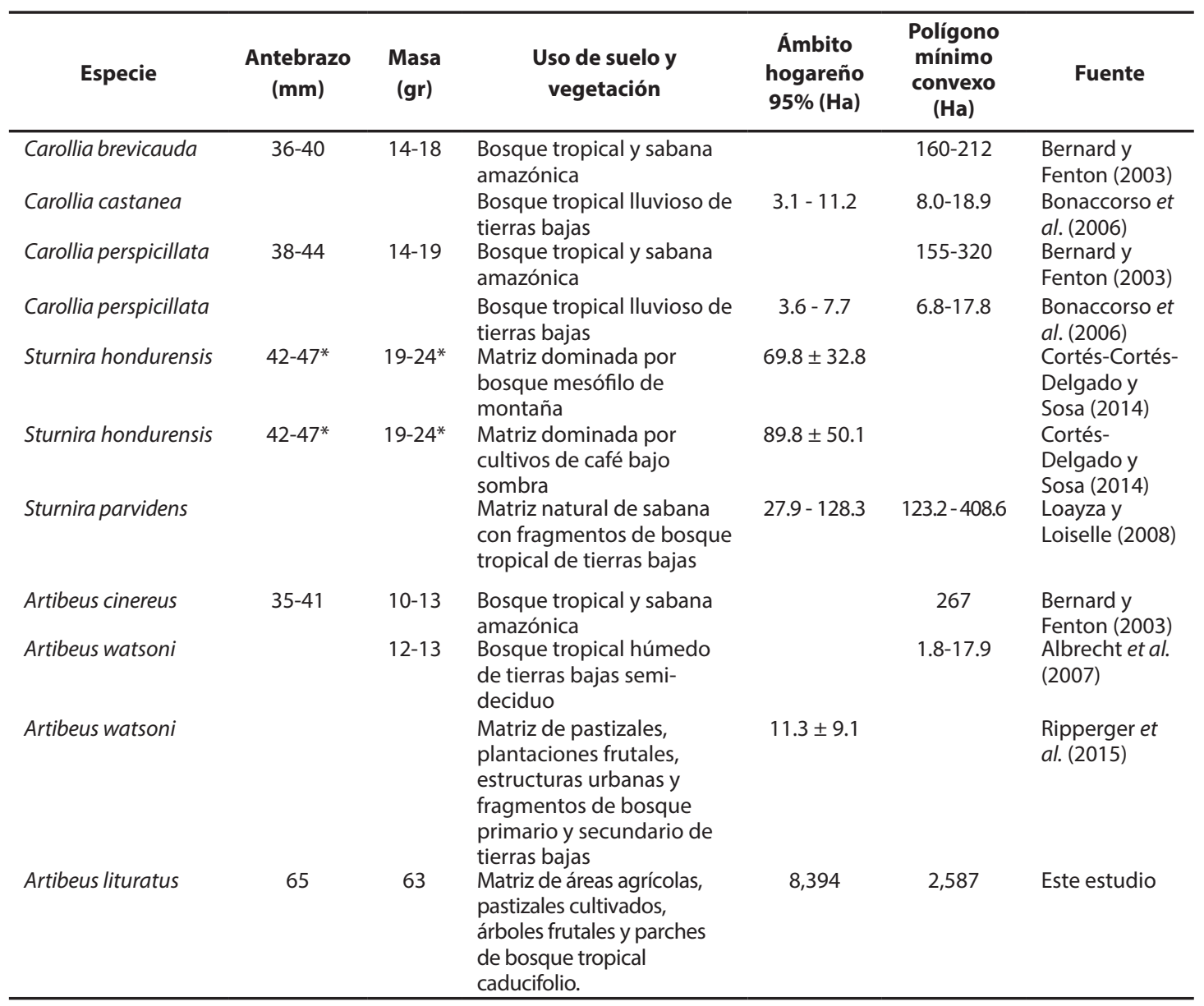

* Tomado de Téllez-Girón (2005) 


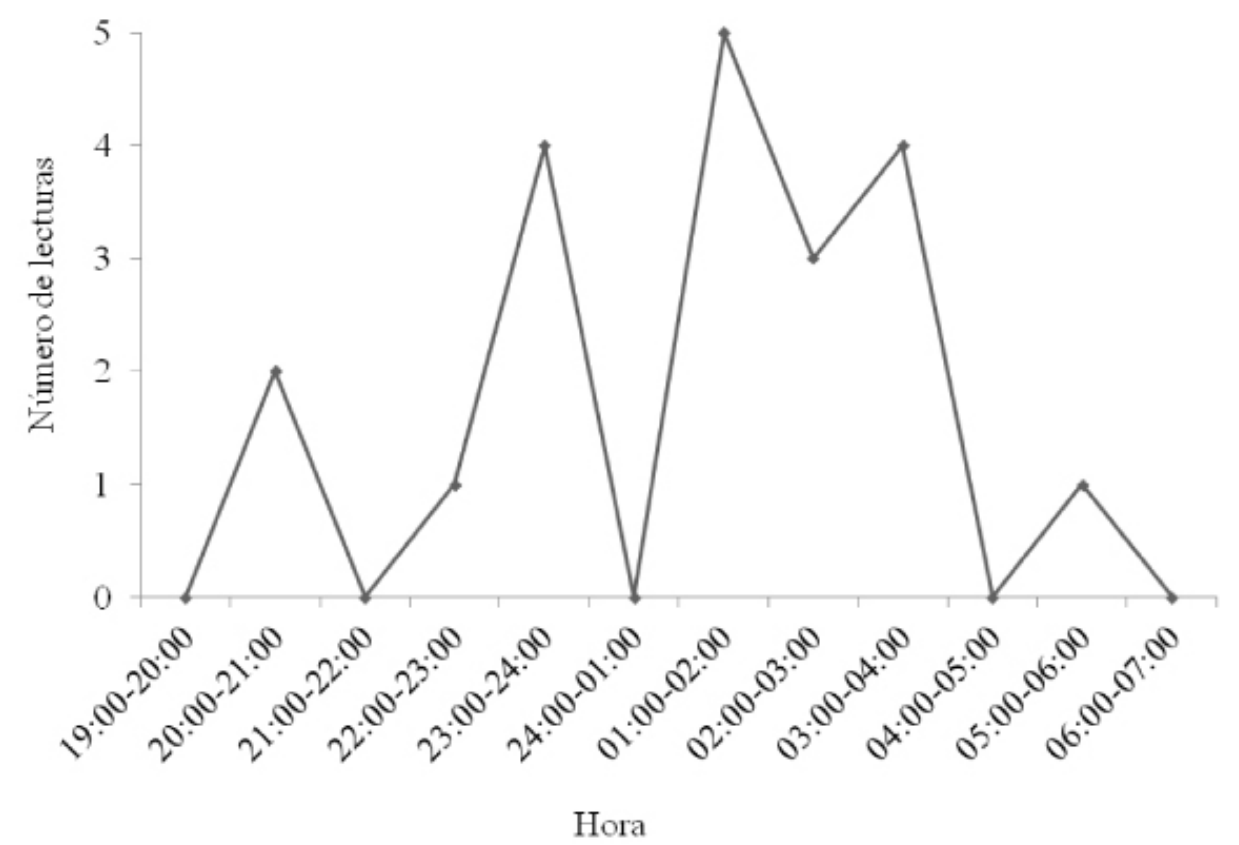

Figura 4. Actividad circadiana observada a traves del total de lecturas del rumbo de tres hembras adultas de Pteronotus parnellii equipadas con radio-transmisores en el Istmo de Tehuantepec.

2003), la estrategia de forrajeo, la dieta, la distribución de los recursos alimenticios y la morfología del ala (Meyer et al. 2005). En el presente estudio, las condiciones del hábitat pueden estar orillando a A. lituratus a ampliar su área de actividad para satisfacer sus necesidades de alimentación, como consecuencia de la baja cobertura arbórea y la presencia de parques eólicos.

A pesar de la baja cobertura arbórea, las áreas agrícolas rodeadas de fragmentos no son una barrera para el movimiento de los murciélagos (Bernard y Fenton 2003), y las cercas vivas pueden servir de vía para el movimiento entre fragmentos (Estrada y Coates-Estrada 2001). Lo que puede explicar la actividad y uso de hábitat en esta zona del Istmo de Tehuantepec de A. lituratus, A. jamaicensis, L. yerbabuenae y P. parnellii.

\section{Agradecimientos}

B. Riveros-Lara, Y. Martínez-Ayón, A G. Monroy-Gamboa y N. Martín-Regalado por su apoyo en la toma de datos. M.B.S. agradece al Sistema de Becas de exclusividad y al programa de Estímulos al Desempeño a la Investigación del Instituto Politécnico Nacional por el apoyo. De igual forma, al Sistema Nacional de Investigadores por el reconocimiento. Dos revisores anónimos, el editor asociado y el editor en jefe realizaron observaciones que ayudaron a mejorar el manuscrito.

\section{Literatura citada}

Aihartza, J. R., I. Garin, U. Goiti, J. Zabala, e I. Zuberogoitia. 2003. Spring habitat selection by the Mediterranean Horseshoe Bat (Rhinolophus euryale) in the Urdaibai Biosphere Reserve (Basque Country). Mammalia 67:25-32.

Albrecht, L., C. F. J. Meyer, y E. K. V. Kalko. 2007. Differential mobility in two small phyllostomid bats, Artibeus watsoni and Micronycteris microtis, in a fragmented neotropical landscape. Acta Theriologica 52:141-149. 
Aldridge, H. D. J. N., y R. M. Brigham. 1988. Load carrying and maneuverability in an insectivorous bat: a test of the $5 \%$ "rule" of radiotelemetry. Journal of Mammalogy 69:379-382.

Álvarez, T., S. T. Alvarez-Castañeda, y J. C. L. Vidal. 1994. Claves para murciélagos mexicanos. Centro de Investigaciones Biológicas del Noroeste. La Paz, Baja California Sur, México.

Arias-Cóyotl, E., K. E. Stoner, y A. Casas. 2006. Effectiveness of bats as pollinators of Stenocereus stellatus (Cactaceae) in wild, managed in situ, and cultivated populations in La Mixteca Baja, Central Mexico. American Journal of Botany 93:1675-1683.

Arnone, I. S., E. Trajano, A. Pulchério-Leite, y F. De C. Passos. 2016. Long-distance movement by a great fruit-eating bat, Artibeus lituratus (Olfers, 1818), in southeastern Brazil (Chiroptera, Phyllostomidae): evidence for migration in Neotropical bats? Biota Neotropica 16:e0026.

Arriaga-Flores, J. C., I. Castro-Arellano, A. Moreno-Valdez, y A. Correa-Sandoval. 2012. Temporal niche overlap of a riparian forest bat assemblage in subtropical Mexico. Revista Mexicana de Mastozoología Nueva época 2:3-17.

Bernard, E., y M. Fenton. 2003. Bat mobility and roosts in a fragmented landscape in Central Amazonia, Brazil. Biotropica 35:262-277.

Bianconi, G. V., S. B. Mikıch, Y W. A. Pedro. 2006. Movements of bats (Mammalian, Chiroptera) in Atlantic Forest remnants in southern Brazil. Revista Brasileira de Zoologia 23:1199-1206.

BonAcCoRso, F. J. 1979. Foraging and reproductive ecology in a Panamanian bat community. Bulletin of the Florida State Museum, Biological Sciences 24:359-412.

Bonaccorso, F. J., J. R. Winkelmann, D. Shin, C. I. Agrawal, N. Aslami, C. Bonney, A. Hsu, P. E. Jekielek, A. K. Knox, S. J. Kopach, T. D. Jennings, J. R. Lasky, S. A. Menesale, J. H. Richards, J. A. Rutland, A. K. Sessa, L. Zhaurova, y T. H. Kunz. 2006. Evidence for exploitable competition: comparative foraging behavior and roosting ecology of short-tailed fruit bats (Phyllostomidae). Biotropica 39:249-256.

Carrillo-Reyes, A., C. Lorenzo, T. Rioja-Paradela, E. Naranjo, y M. Pando. 2012. Uso de hábitat de la liebre en peligro de extinción, Lepus flavigularis: implicaciones para su conservación. Therya 3:113-125.

Cortéz-Delgado, N., y V. J. Sosa. 2014. Do bats roost and forage in shade coffee plantations? A perspective from frugivorous bat Sturnira hondurensis. Biotropica 46:624-632.

Erkert, H. G. 2000. Bats-Flying nocturnal mammals. Pp. 253-272 in Activity patterns in small mammals (Halle, S., y N. C. Stenseth, eds.). Springer-Verlag, Berlin Heidelberg. Berlín, Alemania.

Estrada, A., y R. Coates-Estrada. 2001. Species composition and reproductive phenology of bats in a tropical landscape at Los Tuxtlas, Mexico. Journal of Ecology 17:627-646.

FAHRIG, L. 2003. Effects of habitat fragmentation on biodiversity. Annual Review of Ecology, Evolution, and Systematics 34:487-515.

Fenton, M. B., E. Bernard, S. Bouchard, L. Hollis, D. S. Johnston, C. L. Lausen, J. M. Ratcliffe, D. K. RIskin, J. R. TAYLOR, Y J. ZıgouRIs. 2001. The bat fauna of Lamanai, Belize: roosts and trophic roles. Journal of Tropical Ecology 17:511-524.

Galindo-González, J. 2004. Clasificación de los murciélagos de la región de los Tuxtlas, Veracruz, respecto a su respuesta a la fragmentación del hábitat. Acta Zoológica Mexicana (n. s.) 20:239-243.

García, E. 2001. Climas. Mapa vectorial 1:1 000 000. CONABIO. Ciudad de México, México.

Goiti, U., J. R. Aimartza, I. Garin, y J. Zabala. 2003. Influence of habitat on the foraging behaviour of the Mediterranean Horseshoe Bat, Rhinolophus euryale. Acta Chiropterologica 5:75-84. 
Handley, C. O. JR., y D. W. MorRison. 1991. Foraging behavior. Pp. 137-140 Demography and natural history of the common fruit bat, Artibeus jamaicensis, on Barro Colorado Island, Panama (Handley, Jr., C. O., D. E. Wilson, y A. L. Gardner, eds.). Smithsonian Contributions to Zoology 511. Washington, EE. UU.

Herd, R. M. 1983. Pteronotus parnellii. Mammalian Species 209:1-5.

Hernández-Escobedo, Q., F. Manzano-Agugliaro, y A. Zapata-Sierra. 2010. The wind power of México. Renewable and Sustainable Energy Reviews 14:2830-2840.

Horner, M. A., T. H. Fleming, y C. T. Sahley. 1998. Foraging behavior and energetics of a nectar-feeding bat, Leptonycteris curasoae (Chiroptera: Phyllostomidae). Journal of Zoology 244:575-586.

INEGI. 2005. Mapa de uso de suelo y vegetación serie V. Mapa vectorial, escala 1:250 000. INEGI. San Luis Potosí, México.

Jennings, N. V., S. Parsons, K. E. Barlow, y M. R. Gannon. 2004. Echolocation calls and wing morphology of bats from the West Indies. Acta Chiropterologica 61:75-90.

Kunz, T.H., C. Wemmer, y V. Haysen. 1996. Sex, age and reproductive condition of mammals. Pp. 279-290 in Measuring and monitoring biological diversity, standard methods for mammals (Wilson, D. E., F. R. Cole, J. D. Nichols, R. Rudran, y M. S. Foster, eds.). Smithsonian Press. Washington, EE. UU.

LoAyzA, A.P., Y B.A. LoIselLe. 2008. Preliminary information on the home range and movement patterns of Sturnira lilium (Phyllostomidae) in a naturally fragmented landscape in Bolivia. Biotropica 40:630-635.

Lorenzo, C., E. C. Sántiz, D. A. Navarrete, y J. Bolaños. 2014. Causes and consequences of change rates in the habitat of the threatened tropical porcupine, Sphiggurus mexicanus (Rodentia: Erethizontidae) in Oaxaca, Mexico: implications for its conservation. Revista de Biología Tropical 62:1481-1494.

Medellín, R. A., H. T. Arita, y O. Sánchez. 1997. Identificación de los murciélagos de México, clave de campo. Asociación Mexicana de Mastozoología, A. C. Ciudad de México, México.

Mendes, P., T. B. Vieira, M. Oprea, y A. D. Ditchfield. 2009. Long-distance movement of Artibeus lituratus (Chiroptera: Phyllostomidae) in the State of Espírito Santo, Brazil. Ecotropica 15:43-46.

Meyer, C. F. J., M. Weinbeer, y E. K. V. KalKo. 2005. Home-range size and spacing patterns of Macrophyllum macrophyllum (Phyllostomidae) foraging over water. Journal of Mammalogy 86:587-598.

Molina-Freaner, F., A. Rojas-Martínez, T. H. Fleming, y A. Valiente-Banuet. 2004. Pollination biology of the columnar cactus Pachycereus pecten-aboriginum in north-western México. Journal of Arid Environments 56:117-127.

Montiel, S., A. Estrada, y P. León. 2006. Bat assemblages in a naturally fragmented ecosystem in the Yucatan Peninsula, Mexico: species richness, diversity and spatio-temporal dynamics. Journal of Tropical Ecology 22:267-276.

Nams, V. O. 2000. Locate II. A program to triangulate radiotelemetry bearings and calculate error ellipses. Dalhousie University. Nova Scotia, Canadá.

Ortiz-Pérez, M. A., J. R. Hernández, y J. M. Figueroa. 2004. Reconocimiento fisiográfico y geomorfológico. Pp. 43-54 Biodiversidad de Oaxaca (García-Mendoza, A. J., M. J. Ordóñez, y M. Briones-Salas, eds.). Instituto de Biología, UNAM-Fondo Oaxaqueño para la Conservación de la Naturaleza-World Wildlife Fund. Ciudad de México, México.

Padilla, L. S., y V. Quiroz. 2005. Dinámica territorial urbana en el Estado de Oaxaca 19502000. Polígonos. Revista de Geografía 15:65-87.

Patterson, B. D., M. R. Willing, y R. D. Stevens. 2003. Trophics strategies, niche partitioning, and patterns of ecological organization. Pp. 536-579 Bat ecology (Kunz, T. H., y M. B. Fenton, eds.). University of Chicago Press. Chicago, EE. UU. 
Ripperger, S. P., E. K. V. Kalko, B. Rodríguez-Herrera, F. Mayer, y M. Tschapka. 2015. Frugivorous bats maintain functional habitat connectivity in agricultural landscape but rely strongly on natural forest fragments. PLoS ONE 14:e0120535.

Rogers, A. R., y J. G. KIE. 2007. Home Range Tools for ArcGIS. Centre for Northern Forest Ecosystem Research. Tunder Bay, Canadá.

Rydell, J., H. T. Arita, M. Santos, y J. Granados. 2002. Acoustic identification of insectivorous bats (order Chiroptera) of Yucatan, Mexico. Journal of Zoology 257:27-36.

SENER (Secretaría de Energía). 2015. Inventario Nacional de Energías Renovables (INERE). Disponible en http://inere.energia.gob.mx.

Téllez-GiRón, G. 2005. Sturnira ludovici. Pp. 248-249 in Mamíferos silvestres de México (Ceballos, G., y G. Oliva, eds.). Fondo de Cultura Económica, Comisión Nacional para el Conocimiento y Uso de la Biodiversidad. Ciudad de México, México.

Tibeitts, T., A. Pate, Y. Petryszyn, y B. Barns. 2002. Determining foraging and roosting areas for Underwood's Mastiff Bat (Eumops underwoodi) using radiotelemetry, at Organ Pipe Cactus National Monument, Arizona. Final Summary Report, year two - December 2002. Desert Southwest Cooperative Ecosystems Studies Unit, The University of Arizona, Tucson. Disponible en: https://www.nps.gov/orpi/learn/nature/upload/ EumopsFinal02.pdf

Von Helversen, O., E Y. Winter. 2003. Glossophagine bats and their flowers: costs and benefits for plants and pollinators. Pp. 346-397 in Bat ecology (Kunz, T. H., y M. B. Fenton, eds.). University of Chicago Press. Chicago, EE. UU.

Voigt, C. C., D. H. Kelm, y G. H. Visser. 2006. Field metabolic rates of phytophagus bat: do pollination strategies of plants make life of nectar-feeders spin faster? Journal of Comparative Physiology 176:213-222.

Submited: January 19, 2016

Reviewed: March 7, 2016

Accepted: April 24, 2016

Associated editor: Cristina Macswiney 